\title{
Decision-Making Process in Patients with Thoracolumbar and Lumbar Burst Fractures with Thoracolumbar Injury Severity and Classification Score Less than Four
}

\author{
Shirzad Azhari, Parisa Azimi, Sohrab Shahzadi, Hassan Reza Mohammadi, Hamid Reza Khayat Kashani \\ Department of Neurosurgery, Shahid Beheshti University of Medical Sciences, Tehran, Iran
}

\begin{abstract}
Study Design: Cross-sectional.
Purpose: To develop a strategy to determine a sound method for decision-making based on postoperative clinical outcome satisfaction. Overview of Literature: The ideal management of thoracolumbar and lumbar burst fractures (TLBF) without neurological compromise remains controversial.

Methods: This was a prospective study. Patients with thoracolumbar injury severity and classification score (TLICS) $<4$ were treated nonoperatively, with bed rest and bracing until the pain decreased sufficiently to allow mobilization. Surgery was undertaken in patients with intractable pain despite an appropriate nonoperative treatment (surgery group). The Oswestry disability index (ODI) measure was observed at baseline and at the last follow-up. Clinically success was defined at least a 30\% improvement from the baseline ODI scores in both the conservative and surgery groups. All case records were assessed for gender, age, residual canal and angulations at the site of the fracture in order to determine which patients benefited from surgery or conservative treatment and which did not.

Results: In all 113 patients with T11-L5, TLBFs were treated. The patients' mean age was 49.2 years. Patients successfully completed either nonoperative ( $n=99$ ) or surgical $(n=14)$ treatment based on ODI. Clinical examinations revealed that all of the patients had intact neurology. The mean follow-up period was 29.5 months. There was a significant difference between the two groups based on age and residual canal. The mean ODI score significantly improved for both groups $(p<0.01)$. According to the findings, a decision matrix was proposed.

Conclusions: The findings confirm that TLICS $<4$, age, and residual canal can be used to guide the treatment of TLBF in conservative decision-making.
\end{abstract}

Keywords: Thoracolumbar injury severity and classification score; Nonoperative; Thoracolumbar and lumbar burst fractures; Outcome; Oswestry disability index

\section{Introduction}

Thoracolumbar and lumbar burst fractures (TLBF) are usually related to major trauma and can cause spinal cord damage that results in neural deficits. They account for approximately $15 \%$ of all spinal injuries [1,2]. The ideal

\footnotetext{
Received Apr 7, 2015; Revised May 6, 2015; Accepted May 7, 2015

Corresponding author: Parisa Azimi

Functional Neurosurgery Research Center of Shohada Tajrish Hospital, Shahid Beheshti University of Medical Sciences,

Sharadari St., Tajrish Square, Tehran 1989934148, Iran

Tel: +98-2122749204 Fax: +98-2188265188, E-mail: parisa.azimi@gmail.com
} 
management of TLBF without neurological compromise remains controversial [3]. There are a number of system tools, including the thoracolumbar injury severity and classification score (TLICS) (Table 1) and American Spinal Injury Association (ASIA) Impairment Scale, which have been proposed for TLBF. The primary goal of the system tools is to establish a reliable and reproducible methodology for categorizing these injuries for the decision-making process [4-6]. Nonoperative treatment offers many benefits, including reduced costs and complications with improved quality of life, so the accurate determination of which patients do not require surgery is highly beneficial $[7,8]$. The TLICS score can be effectively used to classify thoracolumbar spine trauma and can predict surgical treatment [9]. In addition, the Oswestry disability index (ODI) is a well-known tool for measuring functionality in patients with low back pain and has been used to measure functionality in these patients [10]. On the other hand, in the study of predictors of success or failure in the nonoperative treatment of TLBF, Hitchon et al. [11] showed that age and residual canal were significantly effective predictors. However, more evidence is needed to further classify the appropriate TLBF for nonoperative treatment to decrease variables that may impact the prognosis [8]. Hence, there is a need for a consensus on the criteria to lead to the appropriate decision-making process. This study aimed to define a decision matrix for surgery or conservative treatment in patients with TLBF based on TLICS $<4$, age, angulation, and residual canal.

\section{Materials and Methods}

\section{Patients and data collection}

This is a prospective, consecutive case series. Between June 2007 and July 2012, a sample of newly diagnosed single level TLBF patients seen at a large teaching hospital in Tehran, Iran, was investigated. The diagnosis of TLBF was made using clinical symptoms, neurological examinations, and imaging studies, including plain radiography, computed tomography (CT), and magnetic resonance imaging (MRI) of the lumbar spine. The TLICS was evaluated [6]. The treatment plan in patients was chosen according to the patient's comprehensive status, including the degree of injury or damage type, age and residual canal, and typical imaging also was performed if needed. There were no limitations on patient selection with re-
Table 1. The thoracolumbar injury severity and classification score system

\begin{tabular}{lc} 
Variable & Points \\
\hline Injury morphology & \\
\hline Compression & 1 \\
\hline Burst & +1 \\
\hline Translation/rotation & 3 \\
\hline Distraction & 4 \\
\hline Neurological status & \\
\hline Intact & 0 \\
\hline Nerve injury & 2 \\
\hline Cord, conus medullaris & \\
\hline Incomplete & 3 \\
\hline Complete & 2 \\
\hline Cauda equina & 3 \\
\hline Posterior ligamentous complex integrity & \\
\hline Intact & 0 \\
\hline Indeterminate & 2 \\
\hline Injured & 3 \\
\hline
\end{tabular}

As reported by Vaccaro et al. [6].

alFor patients with suggested ligamentous injury on short T1 inversion recovery imaging or T2-weighted magnetic resonance imaging.

gard to level of TLBF, age, or other characteristics. Only patients who had TLICS less than 4 and who were alert and cooperative with neurological testing were included in the study. Patients with TLBF were treated nonoperatively, with bed rest and bracing until the pain abated sufficiently to allow mobilization. Compression stockings were used for deep vein thrombosis prophylaxis during bed rest. Due to intractable pain in some of the patients, surgery was undertaken by two surgeons who were all expert, board-certified members (surgery group). The characteristics including age, gender, residual canal, angulation, and body mass index were recorded. Evaluation of the residual canal was performed according to axial CT images. The residual canal was measured as a percentage of the normal canal above and below the fracture [11]. Angulation was recorded between adjacent intact endplates from lateral plain films. Cases were excluded if the patients had severe related injuries in other regions that might interfere with outcome assessments. The patients with osteoporotic fractures or with histories of previous spine surgery were also excluded from this study. 


\section{Additional measurement}

The Iranian version of Oswestry disability index (ODI). This is a measurement of functionality and contains 10 items. The possible score on the ODI ranges from 0 to 50 , summed, which is then and converted into a percentage score. The range of possible percentage values is from 0 to 100 , with higher scores indicating worse conditions. The psychometric properties of the Iranian version of questionnaire have been well documented [12]. The ODI score was measured at admission and at the last follow-up to assess functionality outcomes after treatment.

\section{Successful outcome measurement}

Clinically, success was defined as a $30 \%$ (or more) improvement from the baseline ODI scores in conservative and surgery groups [13]. The reference points for this study were the date of the initial surgery or conservative procedure. The primary end point for the statistical analysis was a follow-up period of at least two years.

\section{Statistical analysis}

Descriptive statistics were used to explore the data. Differences in baseline characteristics were compared between subjects who received surgery versus subjects who received non-surgical treatment. Student's $t$-tests for continuous data and $\chi^{2}$ tests for categorical data was used, while the statistical significance level was defined as $p<0.05$. All statistical analyses were performed using the PASW Statistics ver. 18 (SPSS Inc., Chicago, IL, USA). Based on the treatment received and based on age and residual canal, patients' successful outcomes were cross-checked in order to provide a road map for decision-making in the future.

\section{Ethics}

The Ethics Committee of Shahid Beheshti University of Medical Sciences, Tehran, Iran, approved the study.

\section{Results}

In all, 124 patients underwent conservative treatment initially. Of these, 14 patients (11.3\%) underwent surgery. The remaining 110 patients only underwent conservative treatment. Of these, 11 patients dropped out of the study or were lost during the follow-up, and the remaining 99 patients were ultimately included in the non-surgical, conservative treatment group. The initial TLICS scores were $2(n=70)$ and $1(n=29)$.

Demographic data for the patients with TLBF and their scores on the ODI, the TLICS, and the residual canal are shown in Table 2. The patient ages ranged from 15 to 72 years of age (mean 49.2 years of age). $43.4 \%$ were female. The etiology of the fractures included falls in 39, motor vehicle accidents in 31, and motorcycle accidents in 25 , lifting in 8 , and sports injuries in 10 . The distribution of the 113 level of fractures were as follows: T11 in 3, T12 in 10, L1 in 45, L2 in 29, L3 in 10, L4 in 13, and L5 in 3. All of the patients had a TLICS $<4$ and were initially treated conservatively. The mean length of hospitalization after injury was 4 (standard deviation $[S D]=3$ ) days, ranging from 1 to 11 days. Surgery was undertaken at $3.1(\mathrm{SD}=2.1)$ months after injury, with a range of 1-6 months. The operative treatment of patients was short transpedicle instrumentation, decompression, and fusion. Patients were followed clinically and radiographically for a minimum of 25 months (mean, 29.5 months). In nonoperative group, angulation significantly progressed from admission to the last follow-up $(p<0.001)$. However, in the surgical group, angulation on admission was not significantly improved at the last follow-up $(p<0.38)$ (Table 2).

Clinical examination revealed that all of the patients had intact neurology. In 14 cases, patients were operated on, with TLICS scores of $2(n=11)$ (compression $1+$ burst $1=2)$ and $3(n=3)$ due to intractable pain. They also had vertebral body height losses of more than 50 compared to the vertebra below.

In the 110 patients who only received conservative treatment, there were $9(8.1 \%)$ who did not achieve a $30 \%$ improvement of $19.2 \%$ to $28.1 \%$ in their ODI score at the last follow-up. These patients were satisfied with the conservative treatment and did not require surgery and dropped out of the study due to the failure of treatment based on their respective ODIs.

After conservative treatment, 14 patients had a mean ODI score less than $30 \%$ improved from 45.4 (16.4) to 39.8 (14.3); they then underwent surgery according to their respective clinical needs. The mean ODI score improved from $45.4(\mathrm{SD}=16.4)$ to $14.1(\mathrm{SD}=12.3)$ for the group treated operatively and $42.1(\mathrm{SD}=15.3)$ to 12.4 $(\mathrm{SD}=11.1)$ for the group treated nonoperatively at the last follow-up $(p<0.01)$. In addition, there was a significant 
Table 2. Baseline demographic data and health status measures based on treatment received in patients with thoracolumbar and lumbar burst fracture $(n=113)$

\begin{tabular}{|c|c|c|c|}
\hline \multirow{2}{*}{ Characteristic } & \multicolumn{3}{|c|}{ Treatment received within one years } \\
\hline & Surgery $(n=14)$ & Non-surgery $(\mathrm{n}=99)$ & $p$-value \\
\hline Age (yr) & $33.4(11.4)$ & $51.4(13.5)$ & 0.003 \\
\hline Gender (female, \%) & 42.9 & 43.4 & 0.892 \\
\hline Body mass index $\left(\mathrm{kg} / \mathrm{m}^{2}\right)$ & $27.4(4.2)$ & $31.8(7.2)$ & 0.604 \\
\hline Residual canal at site fracture $(\%)^{a)}$ & $49.4(13.2)$ & $67.4(10.8)$ & 0.001 \\
\hline Admission angulations at site fracture $\left({ }^{o}\right)^{b)}$ & $6.9(12.4)$ & $5.9(9.8)$ & 0.387 \\
\hline Final angulation at site fracture $\left({ }^{\circ}\right)^{b)}$ & $9.6(11.2)$ & $11.3(9.1)$ & 0.125 \\
\hline Length of hospitalization for surgery (day) & $5(4)$ & - & - \\
\hline Follow-up time (mo) & $27.5(5.2)$ & $29.8(6.3)$ & 0.756 \\
\hline Thoracolumbar injury severity and classification score & $2.2(0.03)$ & $1.7(0.01)$ & 0.235 \\
\hline \multicolumn{4}{|l|}{ Oswestry disability index score ${ }^{c)}$} \\
\hline Baseline & $45.4(16.4)$ & $42.1(15.3)$ & 0.228 \\
\hline At last follow-up & $14.1(12.3)$ & $12.4(11.1)$ & 0.125 \\
\hline
\end{tabular}

Values are presented as mean (standard deviation) or number (\%).

${ }^{a}$ Residual canal, evaluation of residual canal was done according to axial computed tomography images. Residual canal was measured as a per-

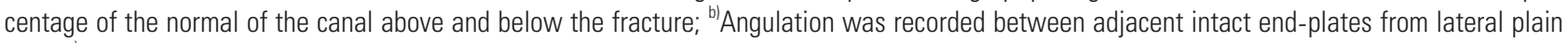
films; ${ }^{\text {cl}}$ The Oswestry disability index ranges from 0 to 100, with lower scores indicating less severe symptoms.

difference based on age (33.4 vs. $51.4 ; p<0.003)$ and residual canal ( 49.4 vs. $67.4 ; p<0.001)$ between the conservative and surgically treated groups, respectively (Table 2).

No patient developed a neurologic deficit, and there were no cases with symptoms indicative of spinal stenosis during the follow-up. In addition, medical complications, such as deep vein thrombosis and pulmonary embolism were not observed in our patients.

Post-treatment satisfaction was observed based on a $30 \%$ improvement in ODI for the group treated operatively and for the group treated nonoperatively. Thus, a decision matrix was derived for choosing the treatment method for these patients (Fig. 1).

\section{Discussion}

Our results established that in patients with TLICS $<4$, age and residual canal jointly are good markers for decisionmaking in TLBF patients.

The TLICS score was designed to assist in the analysis and management of patients with TLBF [6]. This score can help guide treatment decision-making regarding the necessity of surgical or nonsurgical management. Patients with a TLICS of 3 points or fewer are generally treated

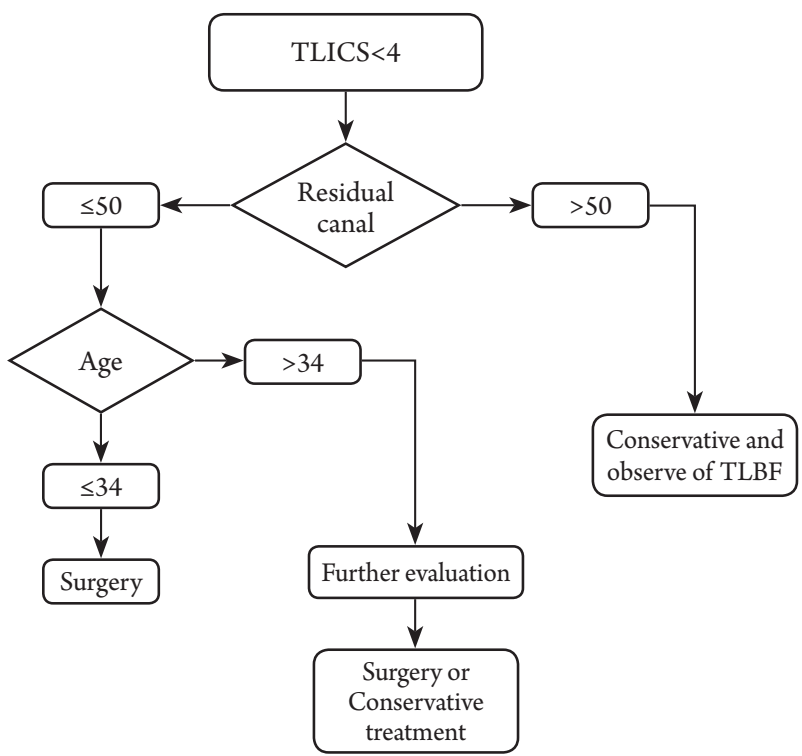

Fig. 1. The algorithm showing the decision-making process for treatment selection in thoracolumbar and lumbar burst fractures. TLICS, thoracolumbar injury severity and classification score; Residual canal, measured as a percentage of the normal of the canal above and below the fracture site according to axial computed tomography images; TLBF, thoracolumbar and lumbar burst fractures.

non-surgically, depending on the type of injury. Patients with a TLICS of 5 or more points generally require surgi- 
cal treatment. Patients with a score of 4 are treated at the discretion of the surgeon [6]. Although, a TLICS score less than 4 can be used to effectively guide conservative treatment [6]. However, in this study, it was found that patients with a TLICS of 3 or less may need surgery if they have intractable pain or if their vertebral body height loss is more than $50 \%$ compared to vertebra below. We think this topic is important because it can help a spine surgeon to make better decisions about treatment plans.

This is the first paper to measure disability in patients with TLBF according to ODI score, TLICS $<4$, age, and residual canal during a follow-up period of at least two years. This study showed that most patients with a TLICS $<4$ might be appropriate candidates for conservative therapy. The findings also showed that patients who failed conservative treatment and ultimately underwent surgery had a mean age of 33.4 years, compared to 51.4 years in the group successfully treated nonoperatively. Also, the residual canal in the operative group was less than that of the nonoperative group, which is line with the findings of other researchers [11].

In the literature review by van der Roer et al. [14], the group reported that studies did not provide a reliable answer to whether operative or conservative treatment was more effective for unstable traumatic thoracolumbar fractures. Additionally, they emphasized that clinical outcome measurements should be included as an evaluation of the success of treatment of the thoracolumbar fractures [14]. Thus, the ODI was applied. A 30\% improvement was considered a useful threshold for identifying clinically meaningful improvement on the ODI in patients with low back pain [13]. Our results show that all of patients who failed conservative treatment and ultimately underwent surgery did not have 30\% improvement on the ODI measure. In addition, 9/110 (8.1\%) patients were successfully treated nonoperatively but did not have a $30 \%$ improvement on the ODI measure at the last follow-up. However, the ODIs at the final follow-up evaluation ranged from 14 to 20 points, which suggests minimal disability [15]. At present, we cannot explain the observed discrepancy, in this study, we were unable to estimate cutoff values to identify patients with successful outcomes after conservative treatment for TLBF according to ODI. However, this study showed that the ODI may be able useful with respect to clinical outcomes in patients with TLBF and TLICS $<4$ at baseline. In our opinion, a cutoff value is needed based on ODI or other exclusive measures for the assessment of successful treatment in these patients.

Burst fractures without neurologic deficit and no injury to the short T1 inversion recovery (STIR) remain a controversial issue [9]. Not all stable burst fractures necessitate surgical management. Wood et al. [16] found no benefit to surgical over nonsurgical treatment of stable burst fractures. Others have also established good to excellent outcomes with the nonsurgical treatment of stable burst fractures, and the TLICS reflects this by also recommending nonsurgical management of these injuries. There is a lack of evidence in studies to resolve this issue and support one approach over the other [9]. In this study, surgical treatment was provided to patients with a TLICS score of 2 and 3 due to intractable pain with decreased vertebral body height. Patients with a TLICS scores of 3 were from compression $1+$ indeterminate $2=3$. Although TLICS did specifically define a T2 signal on STIR as an indeterminate fracture, indeterminate fractures were not observed at the initial evaluation of the 3 patients. Thus, this decision matrix may help clinicians with this issue.

The decision regarding surgery versus conservative treatment should be based on the patient's symptoms, imaging findings, and the spine surgeon's clinical judgment. Considering the fact that a proper guideline for choosing the treatment method for patients with TLBF based on a decision-making tree has not been suggested, we attempted to devise such a flowchart (Fig. 1). It aids the patient and the surgeon during the shared decision-making process. We feel that, because of our study limitations, this algorithm is still primitive and needs to be re-evaluated and modified based on future research efforts. In addition, many other factors from typical imaging studies should be considered in the decision to perform surgery.

There are several principle weaknesses for this study. First, most published studies contain heterogeneous groups of patients, fracture patterns, levels of fractures, number of fractures per patient, neurological statuses, treatment approaches, and outcome tools, which are the main reasons for the continuing controversy [17]. Thus, there is a strong need for improved clinical research methodology to be used to this patient population. Second, we were unable to evaluate all medical interventions and there is no agreement on the optimal method of nonoperative treatment. Hence, further studies investigating such data are recommended. Third, the optimum nonoperative treatment cannot be determined from this study. Fourth, the thoracolumbar classification of fractures is 
typically T10/11 to L2/3, and L4 and L5 fractures as these are biomechanically and structurally different. Hence, this issue should be considered in future studies. Fifth, the question arises, what would have happened to the surgical group if they were allowed to continue on to heal? If the patients in that group were actually delayed healers due to more muscle mass then they received an unnecessary surgery. This is suggested by the randomized control studies on this topic. Sixth, there were 9 patients in the study who did not have $30 \%$ improvement of pain based on ODI and who did not require surgery. These patients were excluded from the study. This may result in a potential bias for the results and undermines the study's conclusions. Seventh, the populations of the nonoperative $(n=99)$ and operative $(n=14)$ groups is vastly different. Hence, further study is needed. Finally, the study had a relatively short followup time. Long-term clinical outcomes, and prospective, randomized studies of nonoperative treatment of TLBF, with standardized definitions of complications will aid in properly defining the risk-benefit ratio of nonoperative treatment of TLBF [18]. The findings of the present study, though, do provide a roadmap to guide future research relating to the decision-making process.

\section{Conclusions}

The findings confirm that for TLICS $<4$, age and residual canal can be used for guiding the treatment of TLBF in conservative decision-making.

\section{Conflict of Interest}

No potential conflict of interest relevant to this article was reported.

\section{Acknowledgments}

The authors thank the staff of the Neurosurgery Unit at Imam-Hossain Hospital, Tehran, Iran.

\section{References}

1. Heary RF, Kumar S. Decision-making in burst fractures of the thoracolumbar and lumbar spine. Indian J Orthop 2007;41:268-76.

2. Zhao X, Fang XQ, Zhao FD, Fan SW. Traumatic canal stenosis should not be an indication for surgical decompression in thoracolumbar burst fracture. Med Hypotheses 2010;75:550-2.

3. Thomas KC, Bailey CS, Dvorak MF, Kwon B, Fisher C. Comparison of operative and nonoperative treatment for thoracolumbar burst fractures in patients without neurological deficit: a systematic review. J Neurosurg Spine 2006;4:351-8.

4. Gummerson NW, Cole AA. Spinal fractures in adults. Surgery 2009;27:287-91.

5. Kirshblum SC, Burns SP, Biering-Sorensen F, et al. International standards for neurological classification of spinal cord injury (revised 2011). J Spinal Cord Med 2011;34:535-46.

6. Vaccaro AR, Lehman RA Jr, Hurlbert RJ, et al. A new classification of thoracolumbar injuries: the importance of injury morphology, the integrity of the posterior ligamentous complex, and neurologic status. Spine (Phila Pa 1976) 2005;30:2325-33.

7. Abudou M, Chen X, Kong X, Wu T. Surgical versus non-surgical treatment for thoracolumbar burst fractures without neurological deficit. Cochrane Database Syst Rev 2013;6:CD005079.

8. Bakhsheshian J, Dahdaleh NS, Fakurnejad S, Scheer JK, Smith ZA. Evidence-based management of traumatic thoracolumbar burst fractures: a systematic review of nonoperative management. Neurosurg Focus 2014;37:E1.

9. Joaquim AF, Fernandes YB, Cavalcante RA, Fragoso RM, Honorato DC, Patel AA. Evaluation of the thoracolumbar injury classification system in thoracic and lumbar spinal trauma. Spine (Phila Pa 1976) 2011;36:33-6.

10. National Council for Osteopathic Research. Oswestry disability index (ODI) [Internet]. London: NCOR; [cited 2015 Sep 2]. Available from: http://www.ncor. org.uk/wp-content/uploads/2012/12/OswestryDisability-questionnairev2.pdf.

11. Hitchon PW, He W, Viljoen S, et al. Predictors of outcome in the non-operative management of thoracolumbar and lumbar burst fractures. Br J Neurosurg 2014;28:653-7.

12. Mousavi SJ, Parnianpour M, Mehdian H, Montazeri A, Mobini B. The Oswestry Disability Index, the Roland-Morris Disability Questionnaire, and the Quebec Back Pain Disability Scale: translation and validation studies of the Iranian versions. Spine (Phila Pa 1976) 2006;31:E454-9. 
13. Ostelo RW, Deyo RA, Stratford P, et al. Interpreting change scores for pain and functional status in low back pain: towards international consensus regarding minimal important change. Spine (Phila Pa 1976) 2008;33:90-4.

14. van der Roer N, de Lange ES, Bakker FC, de Vet HC, van Tulder MW. Management of traumatic thoracolumbar fractures: a systematic review of the literature. Eur Spine J 2005;14:527-34.

15. Fairbank JC, Couper J, Davies JB, O’Brien JP. The Oswestry low back pain disability questionnaire. Physiotherapy 1980;66:271-3.

16. Wood K, Buttermann G, Mehbod A, Garvey T, Jhanjee R, Sechriest V. Operative compared with nonop- erative treatment of a thoracolumbar burst fracture without neurological deficit: a prospective, randomized study. J Bone Joint Surg Am 2003;85:773-81.

17. Koller H, Acosta F, Hempfing A, et al. Long-term investigation of nonsurgical treatment for thoracolumbar and lumbar burst fractures: an outcome analysis in sight of spinopelvic balance. Eur Spine J 2008;17:1073-95.

18. Ghobrial GM, Maulucci CM, Maltenfort M, et al. Operative and nonoperative adverse events in the management of traumatic fractures of the thoracolumbar spine: a systematic review. Neurosurg Focus 2014;37:E8. 